\title{
ANDES

www.scielo.cl

\section{Coocurrencia de trastorno del espectro autista y disforia de género en la infancia: análisis de la identidad personal desde un enfoque psicodinámico}

\section{Cooccurrence of autism spectrum disorder and gender dysphoria in childhood: analysis of the personal identity from a psychodynamic perspective}

\author{
Francisca Valdés R. ${ }^{\mathrm{a}}$, Bernardo Pacheco P. ${ }^{\mathrm{b}}$, Paula Bedregal G. ${ }^{\mathrm{c}}$ \\ aUnidad de Salud Mental, Hospital Clínico Dra. Eloísa Diaz La Florida. Santiago, Chile. \\ bDepartamento de Psiquiatría, Escuela de Medicina, Pontificia Universidad Católica de Chile. Santiago, Chile. \\ ‘Departamento de Salud Pública, Escuela de Medicina, Pontificia Universidad Católica de Chile. Santiago, Chile.
}

Recibido: 8 de septiembre de 2020; Aceptado: 22 de junio de 2021

¿Qué se sabe del tema que trata este estudio?

En la práctica clínica pediátrica se ha detectado un incremento de consultas de pacientes con coocurrencia de TEA y DG. Sin embargo, los estudios que abordan el tema desde una perspectiva de interacción psicopatológica que analice ambas condiciones, son escasos.
¿Qué aporta este estudio a lo ya conocido?

Este estudio, ilustrado por medio de un caso clínico, pone énfasis en la interacción dinámica que ocurre entre el desarrollo de la identidad personal y de género en condiciones psicopatológicas autísticas.

\section{Resumen}

El Trastorno del Espectro Autista (TEA) es una condición del neurodesarrollo con alteraciones en la comunicación e interacción social y comportamientos e intereses repetitivos y restringidos. La Disforia de Género (DG) es la incongruencia entre el sexo que se siente y el asignado al nacer, con deseo de ser del sexo opuesto. El desarrollo de la identidad sexual es un elemento esencial en la construcción de la identidad global individual. Objetivo: Analizar un caso clínico de coocurrencia de TEA y DG en un adolescente desde una comprensión psicodinámica de su identidad personal. Caso Clínico: Paciente de sexo biológico masculino de 15 años diagnosticado con TEA y capacidad intelectual limítrofe. Desde la etapa preescolar manifestó conductas femeninas e intereses por juegos de niñas con imitación de figuras femeninas, verbalizando querer ser del sexo opuesto. Una evaluación multidisciplinaria en la adolescencia concluyó que presentaba una identidad general difusa, con identidad de género ambivalente, por lo que se determinó que no era candidato a terapia hormonal. Se sugirió iniciar proceso terapéutico que favoreciera una mayor exploración y flexibilidad cognitiva para contribuir al
Palabras clave: Identidad; Identidad de Género; Disforia de Género; Trastorno del Espectro Autista 
desarrollo de una identificación de género más definida. Conclusiones: En condiciones de adversidad identitaria, como las presente en el TEA, el sujeto afectado con frecuencia retrasa o bien, no consolida la identidad sexual, coexistiendo una difusión de identidad y de género como en el caso reportado. La coocurrencia de TEA y DG requiere de evaluación multidisciplinaria, incluyendo evaluación completa de la identidad global para definir la orientación terapéutica definitiva respecto del género.

\begin{abstract}
Autism Spectrum Disorder (ASD) is a neurodevelopmental condition with alterations in social interaction and communication, and restricted and repetitive patterns of behavior and interests. Gender Dysphoria (GD) refers to the incongruence between the gender identity and the sex assigned at birth, together with a strong and persistent desire to be of the opposite sex. During global personal identity construction, the development of sexual identity is essential. Objective: To analyze an adolescent clinical case of co-occurrence of ASD and GD from a psychodynamic understanding of his identity. Clinical Case: A 15-year-old male diagnosed with ASD and borderline intellectual capacity. He manifested feminine behaviors and interests in girlish games since preschool age, imitating female figures, and verbalizing the desire to be of the opposite sex. A multidisciplinary evaluation of the patient concluded that he presented a diffuse general identity with ambivalent gender identity. Therefore, he did not fulfill the requirements for hormone therapy. Psychological therapy was suggested to favor greater exploration and cognitive flexibility to develop a better-defined gender identity. Conclusions: In cases as ASD, where identity development is compromised, the affected subject often delays or does not consolidate sexual identity, leading to a diffusion of identity and gender, as in the reported case. The co-occurrence of ASD and GD requires a multidisciplinary evaluation that includes a complete assessment of the patient's global identity before considering any definitive therapeutic orientation regarding gender.
\end{abstract}

Keywords:

Identity;

Gender Identity; Gender Dysphoria; Autism Spectrum Disorder

\section{Introducción}

En los últimos años diversos estudios han reportado una alta coocurrencia pediátrica de Trastorno del Espectro Autista (TEA) y disconformidad o Disforia de Género (DG) ${ }^{1-6}$. El TEA es una condición del neurodesarrollo que clínicamente se expresa por alteraciones en la comunicación e interacción social y comportamientos restringidos y repetitivos ${ }^{7}$, cuya prevalencia se estima en aproximadamente $1,5 \%{ }^{8}$. La DG se refiere a la incomodidad o malestar causado por la discrepancia entre la identidad de género y el sexo asignado a la persona al nacer, y el rol de género asociado y/o las características sexuales primarias y secundarias ${ }^{9}$. La variabilidad de género o género no conforme se refiere al grado en que la identidad, el rol o la expresión de género difieren de las normas culturales prescritas para personas de un sexo en particular ${ }^{9,10}$. La prevalencia estimada de DG en general en adultos es de 4,6 en $100.000^{11}$.

De acuerdo a Kernberg ${ }^{12}$, la identidad personal es el constructo que permite comprender cómo la persona crea, organiza y mantiene de modo cohesionado un sentido de sí mismo (self) diferente de un otro, llevado a cabo por medio de un proceso permanente de interacción social y de construcción de significados. La identidad se organiza en múltiples dimensiones (género, etnia/raza, sexualidad, clase social, cultura) que se intersectan y llegan a constituir finalmente la identidad del sí mismo, vivida como una globalidad y como algo único ${ }^{13}$. La dimensión de identidad de género expresa el sentido personal que cada individuo le da a su propio género basado en su propia experiencia y sentido de $\operatorname{ser}^{14}$. Puede ser consistente o diferente al sexo biológico asignado al nacer. Aquellas expresiones e identidades que difieren de lo binario se describen dentro de un espectro de fluidez de género o género no binario. La identidad de género es un proceso de desarrollo temprano, observándose conductas identificatorias ya a los 2 años de vida en población general ${ }^{15}$. En niños con TEA, los estudios han demostrado que la capacidad de identificar el género se asocia con la edad mental y cronológica ${ }^{16}$.

Se ha reportado alrededor del 5\% de variabilidad de género en niños y adolescentes con TEA expresado como "deseo de ser del sexo opuesto" en la CBCL (Child Behavior Checklist) ${ }^{1-3}$. En niños, niñas y adolescentes con DG se ha detectado una sobrerrepresentación de TEA en comparación con la población gene$\mathrm{ral}^{4-6}$. Algunos estudios consideran las expresiones de género opuesto como un interés obsesivo y restringido del TEA ${ }^{17-19}$, mientras que otros proponen una presentación simultánea, relacionadas estrechamente en sus dimensiones biológicas, sociales y cognitivas ${ }^{20,21}$. 
El objetivo de este trabajo es presentar el caso de un adolescente con TEA y DG, discutiendo el rol que juega la identidad personal en ambas condiciones. Esta publicación cuenta con la aprobación del Comité de Ética, el asentimiento del paciente y el consentimiento de sus padres. Para resguardo de la identidad, se ha usado un nombre ficticio.

\section{Caso Clínico}

Javier, de 15 años y sexo biológico masculino, es hijo menor de una familia nuclear biparental con dos hermanas independientes de 26 y 25 años. Su padre es militar jubilado y su madre dueña de casa, quien tiene el antecedente de un Trastorno del Lenguaje y de un Trastorno por Déficit Atencional, ambos tratados. Javier nació de un embarazo no programado, controlado y de término. Su parto fue por cesárea, sin complicaciones y con parámetros antropométricos normales. Recibió lactancia materna los primeros 5 meses. Presentó retraso en el desarrollo psicomotor, logrando la marcha autónoma a los 15 meses y adquiriendo sus primeras palabras a los 2 años. Logró control de esfínter adecuado. Presentó otitis media a repetición con conservación de la audición. Durante su infancia requirió hospitalizaciones por bronquitis y por cirugía de hernia inguinal y fimosis.

Ingresó al jardín infantil a los 2 años. A los 3 años persistió con retraso en su lenguaje, agregándose inquietud, pensamientos obsesivos, insistencia en rutinas y desinterés por pares. A los 4 años se solicitó una evaluación por Fonoaudiología, Psicología y Terapia Ocupacional, describiéndose un lenguaje comprensivo y expresivo bajo lo esperado para su edad, con nivel pragmático descendido, baja intención comunicativa y contacto ocular escaso, juego solitario y poco flexible. Se diagnosticó un Trastorno del Lenguaje comprensivo-expresivo con un desarrollo psicomotor disarmónico y dificultades de integración sensorial, sospechándose además, un Trastorno Generalizado del Desarrollo. Inició terapia psicológica y ocupacional.

A los 5 años comenzó con conductas obsesivas, autoagresiones y tics motores, indicándose uso de risperidona. En ese entonces, por motivos laborales del padre, la familia se trasladó a Alemania donde residieron durante un año y medio. La sintomatología en Javier se intensificó y aparecieron juegos estereotipados de imitación de figuras femeninas y referencias a sí mismo como "Javier es una niña". Se evaluó clínicamente y con las pruebas ADOS (Autism Diagnostic Observational Schedule) y ADI-R (Autism Diagnostic Interview-Revised), y se diagnosticó Autismo Infantil (CIE-10). La prueba SON-R (Snijders-Oomen Nonverbal Intelligence Test) informó una inteligencia bajo la media. Recibió apoyo terapéutico uno a uno para la sintomatología autista durante los siguientes 14 meses, mostrando mejoría parcial de la conducta, persistiendo con comportamientos de tendencia femenina.

$\mathrm{Al}$ regreso al país, Javier ingresó al sistema escolar contando con apoyo psicopedagógico y de salud mental, pero persistió con dificultades académicas y en la interacción con pares. A los 10 años se realizó una reevaluación neuropsicológica con pruebas WISC-III (Wechsler Intelligence Scale for Children-Third Edition) y CAT-A (Children's Aperception Test - Animals) que informaron una capacidad intelectual limítrofe disarmónica y un desarrollo alterado de la estructura de personalidad. Dada sus dificultades de ajuste conductual y académico, recibió farmacoterapia con metilfenidato, fluoxetina y aripiprazol, respondiendo parcialmente.

Las conductas de orientación femenina continuaron en el paciente con intensidad variable según la aceptación y tolerancia del entorno. En los dibujos solo hacía figuras femeninas, descritas como "bellas y preocupadas de verse bonitas", también se dibujaba a sí mismo con cuerpo femenino. Mostraba gran atracción a accesorios como aros, pintura de uñas y vestuario femenino, recurría frecuentemente a fantasías femeninas y temas relacionados con las mujeres. En el juego tomaba roles femeninos de "ayudanta" o bailarina, se pintaba los labios, modelaba y pintaba. A los 11 años manifestaba sus intereses como "me gusta coser, cocinar, diseñar modas y vestuario". A los 15 años ocasionalmente se hacía llamar "Alicia". Estas conductas eran observadas principalmente en el ambiente familiar.

Durante este periodo el paciente se mantuvo con terapia farmacológica y psicoeducación a los padres respecto al apoyo escolar y manejo conductual para la evitación de riesgos y de transgresiones. En una ocasión fue sorprendido por los padres haciendo uso de tarjetas de crédito para compras por internet de muñecas. Algunas de estas conductas no tuvieron una adecuada aceptación por algunos parientes, generando agresión y maltrato. Los padres denunciaron esta situación. La familia ha recibido gran apoyo de sus cercanos en estas crisis familiares, pero también han implicado quiebres de algunas relaciones en la familia extendida. Javier recibió terapia psicológica breve buscando mejorar su comportamiento y orientar sus conductas femeninas hacia un ajuste social menos disruptivo, con escasos resultados

$\mathrm{Al}$ entrar en la adolescencia, Javier presentó un deterioro auditivo progresivo idiopático que requirió uso permanente de audífonos. En el mismo periodo se realizó una evaluación endocrinológica y genética, con el objetivo de reevaluar el caso y reorientar la terapia. Los resultados dieron cuenta de un examen físico sin dismorfias, detectándose una obesidad con IMC $33 \mathrm{Kg} / \mathrm{m}^{3}$. Su 
desarrollo puberal se encontraba en Tanner IV. El estudio con cariograma, PCR para X Frágil y Array CGH, descartó patología genética concomitante. Esta reevaluación se complementó con la observación de un equipo de Salud Mental especialista en identidad género. Los profesionales opinaron que dado el funcionamiento infantil, el desarrollo intelectual del paciente y la escasa angustia relacionada a la disforia, no era candidato por el momento a una terapia de género hormonal ya que se encontraba dentro de un estado de "fluidez de género" con una identidad general dispersa. Se sugirió iniciar un proceso terapéutico que favoreciera en el paciente una mayor exploración y flexibilidad cognitiva de sus esquemas mentales de pertenencia a un determinado género, para así ayudar al logro de una identificación de género más consistente y definida.

\section{Discusión}

El seguimiento a largo plazo realizado en el paciente ha permitido observar las manifestaciones clínicas de la coocurrencia de TEA y DG, interrogando al clínico sobre cómo esta coexistencia puede tener efecto en el desarrollo y construcción de la identidad personal del sujeto.

La identidad personal se construye durante la infancia y adolescencia mediante un proceso permanente de interacción social y de construcción de significados. Comprendemos la identidad de género como un componente de la identidad sexual global, siendo esta última uno de los elementos constitutivos de la identidad personal total del sujeto, como lo es también, entre otros, el temperamento, los afectos y la cognición ${ }^{12}$. De acuerdo a la teoría contemporánea de relaciones objetales, una identidad normal debe lograr la integración del self y de las representaciones de los otros significantes ${ }^{22}$.

De acuerdo con Kernberg se identifican cuatro factores de la conducta sexualizada individual constitutivas de la identidad de género: la identidad genérica nuclear (el individuo se considera hombre o mujer); la identidad de rol genérico (actitudes psicológicas y conductas interpersonales); la elección de objeto dominante (selección de objeto sexual, heterosexual u homosexual) y la intensidad del deseo sexual (fantasía sexual $)^{23,24}$. En nuestro paciente se ha podido evaluar, por medio de la entrevista y el comportamiento, que los dos primeros factores, género nuclear y rol de género, no parecen consolidados ni integrados, lo cual puede debilitar no sólo la identidad de género, sino también la identidad global del paciente, ya interferida por el TEA. La relevancia clínica está en el hecho de que ambos componentes, la identidad (conciencia de quién se es), y el género (la conciencia de ser varón o mujer), funcionan evolutivamente como elementos constitutivos e interactivos de la formación de la personalidad global del sujeto ${ }^{12}$. En el caso presentado pensamos que ambas condiciones, DG y TEA, al coocurrir simultáneamente interfieren sinérgicamente alterando el desarrollo de la identidad global y, por ende, de la personalidad del paciente.

En el desarrollo psíquico es necesario que la integración sana de la personalidad vaya adherida a una adecuada construcción de la identidad genérica nuclear ${ }^{23}$. Por lo tanto, favorecer el desarrollo de una identidad de género armónica en nuestro paciente juega un rol constitutivo fundamental del núcleo de identidad yoica de su personalidad. Dicho de otro modo, la falta de integración de la identidad global ya presente en el TEA puede ser afectada negativamente y empeorada por una "difusión de identidad sexual". La "difusión sexual" (identidad sexual en desarrollo poco diferenciada y constituida frágilmente) de nuestro paciente se suma a la falta de integración identitaria, conocida psicopatológicamente como difusión de identidad, condición de estructura mental que puede estar presente en personas con TEA. Clínicamente la difusión de identidad se expresa ante el entrevistador como una dificultad de la persona para dar cuenta de quién sé es (un self integrado) y de los otros de manera integrada, con un funcionamiento interpersonal inestable ${ }^{25}$.

Sumado a lo anterior, debemos tener en cuenta que las dificultades intelectuales que presentan algunas personas con TEA exacerban la rigidez y dispersión cognitiva, contribuyendo negativamente a la claridad de pertenencia a un determinado género. Si bien nuestro paciente manifiesta un interés por el género femenino, es posible que la difusión de identidad global sumado a las dificultades intelectuales que presenta (capacidad intelectual limítrofe disarmónica) estén limitando el avance hacia una mayor estructuración de su identidad de género. Este aspecto se manifiesta clínicamente al interrogar al paciente respecto a su autoimagen, a su sentir y a su orientación de género, donde sus respuestas no reflejan una disforia por lo masculino y sólo expresan deseos concretos de obtener objetos de connotación femenina, elegidos más por su apariencia que por un sentido de representación mental de esos objetos en un sí-mismo identificado con un determinado género, dando cuenta de este modo sólo de aspectos muy parciales de su identidad global.

Finalmente, considerando que las personas con TEA pueden presentar dificultades de comunicación y menor habilidad para hacer registros de sí mismos, la elección de las técnicas terapéuticas orientadas a la estimulación de una identificación sexual más integrada deben evitar imponer una orientación de género determinada, sino más bien orientar la ayuda a lo que el paciente es capaz de descubrir, construir y desarrollar 
como personalidad, buscando integrar tanto aspectos de la imagen de sí mismo como de su sexualidad. Una implementación no oportuna de las intervenciones para adolescentes podría prolongar la DG y contribuir a abusos y estigmatismos ${ }^{9}$.

\section{Conclusiones}

El trabajo oportuno, colaborativo y de comprensión multidimensional entre especialistas en personas con coocurrencia de TEA y DG permite evaluar tempranamente tanto el desarrollo de la identidad de género como el de su identidad global. Si bien existen diversas comprensiones de la coocurrencia de TEA y DG, contar con un marco teórico comprensivo psicodinámico que analice el rol integrador que juega la identidad sexual en la construcción de la identidad global del paciente y, por ende, de su personalidad, ayuda a una compresión clínica de la coocurrencia y orienta el sentido de las intervenciones terapéuticas.

\section{Responsabilidades Éticas}

Protección de personas y animales: Los autores declaran que los procedimientos seguidos se conformaron a las normas éticas del comité de experimentación humana responsable y de acuerdo con la Asociación Médica Mundial y la Declaración de Helsinki.
Confidencialidad de los datos: Los autores declaran que han seguido los protocolos de su centro de trabajo sobre la publicación de datos de pacientes.

\section{Derecho a la privacidad y consentimiento informa-} do: Los autores han obtenido el consentimiento informado de los pacientes y/o sujetos referidos en el artículo. Este documento obra en poder del autor de correspondencia.

\section{Conflicto de intereses}

Los autores declaran no tener conflicto de intereses.

\section{Agradecimientos}

Agradecemos la constante cooperación y disposición de los padres y la familia nuclear del paciente, y destacamos su esfuerzo y tenacidad en velar por el mayor bienestar de su hijo, pese a las dificultades y adversidades que han tenido en este camino. Agradecemos, también, la colaboración del Programa Grupo Apoyo a Diversidad Sexual (GADi) de la Red UC-Christus del Centro Médico San Joaquín en la evaluación clínica del paciente, como así también la participación de la Dra. Cecilia Mellado y el Dr. Alejandro Martínez, genetista y endocrinólogo pediátrico, respectivamente, de la Red UC - Christus.

\section{Referencias}

1. Strang JF, Kenworthy L, Dominska A, et al. Increased Gender Variance in Autism Spectrum Disorders and Attention Deficit Hyperactivity Disorder. Arch Sex Behav. 2014;43(8):1525-33.

2. Janssen A, Huang H, Duncan C. Gender Variance Among Youth with Autism Spectrum Disorders: A Retrospective Chart Review. Transgender Heal. 2016;1(1):63-8.

3. May T, Pang K, Williams KJ. Gender variance in children and adolescents with autism spectrum disorder from the National Database for Autism Research. Int J Transgenderism. 2017;18(1):7-15.

4. de Vries ALC, Noens ILJ, CohenKettenis PT, et al. Autism spectrum disorders in gender dysphoric children and adolescents. J Autism Dev Disord. 2010;40(8):930-6.

5. Skagerberg E, Di Ceglie D, Carmichael P. Brief Report: Autistic Features in Children and Adolescents with Gender Dysphoria. J Autism Dev Disord. 2015;45(8):2628-32.
6. van der Miesen AIR, de Vries ALC, Steensma TD, et al. Autistic symptoms in children and adolescents with gender dysphoria. J Autism Dev Disord. 2017;48(5):1537-48.

7. American Psychiatric Association. Guía de consulta de los criterios diagnósticos del DSM- ${ }^{\circledR}$. Guía de consulta de los criterios diagnósticos del DSM- ${ }^{\circledR}$. American Psychiatric Publishing; 2013.

8. Lord C, Elsabbagh M, Baird G, et al. Autism spectrum disorder. Lancet. 2018;392(10146):508-20.

9. The World Professional Association for Transgender Health. Standards of Care for the Health of Transsexual, Transgender, and Gender Nonconforming People, 7th version. WPATH. 2012.

10. Adelson SL, American Academy of Child and Adolescent Psychiatry (AACAP) Committee on Quality Issues (CQI). Practice parameter on gay, lesbian, or bisexual sexual orientation, gender nonconformity, and gender discordance in children and adolescents. J Am Acad Child Adolesc Psychiatry.
2012;51(9):957-74.

11. Arcelus J, Bouman WP, Van Den Noortgate W, et al. Systematic review and meta-analysis of prevalence studies in transsexualism. Eur Psychiatry. 2015;30(6):807-15.

12. Kernberg PF, Weiner AK, Bardenstein KK. Capítulo 2: Componentes de la personalidad. En: Trastornos de la personalidad en niños y adolescentes. $1^{\text {a }}$ ed. Editorial Manual Moderno; 2002.

13. Otis M \& Harley D. Capítulo 5: The intersection of identities of LGBT elders: race, age, sexuality, and care network. En: Handbook of LGBT Elders: An Interdisciplinary Approach to Principles, Practices, and Policies. Eds. Harley DA, Teaster PB. Ed. Springer International Publishing; 2016.

14. Cooper K, Smith LGE, Russell AJ. Gender Identity in Autism: Sex Differences in Social Affiliation with Gender Groups. J Autism Dev Disord. 2018;48(12):39954006.

15. Martin CL, Ruble DN, Szkrybalo J. Cognitive theories of early 
gender development. Psychol Bull. 2002;128(6):903-33.

16. Abelson AG. The development of gender identity in the autistic child. Child Care Health Dev. 1981;7(6):347-56.

17. Gallucci G, Hackerman F, Schmidt CW. Gender Identity Disorder in an Adult Male with Asperger's Syndrome. Sex Disabil. 2005;23(1):35-40.

18. Williams PG, Allard AM, Sears L. Case study: Cross-gender preoccupations in two male children with autism. J Autism Dev Disord. 1996;26(6):635-42.
19. Kraemer B, Delsignore A, Gundelfinger R, et al. Comorbidity of Asperger syndrome and gender identity disorder. Eur Child Adolesc Psychiatry. 2005;14(5):292-6.

20. Landén M, Rasmussen P. Gender identity disorder in a girl with autism: A case report. Eur Child Adolesc Psychiatry. 1997;6(3):170-3.

21. Mukaddes NM. Gender identity problems in autistic children. Child Care Health Dev. 2002;28(6):529-32.

22. Kernberg OF. Identity: Recent Findings and Clinical Implications. Psychoanal Q.
2006;75(4):969-1004.

23. Kernberg OF. Capítulo 1: La experiencia sexual. En: Relaciones amorosas : normalidad y patología. $1^{\mathrm{a}} \mathrm{ed}$. Editorial Paidós; 1995.

24. Tyson P, Tyson RL. Psychoanalytic theories of development: an integration. Yale University Press; 1990.

25. Kernberg OF. Capítulo 1: Diagnóstico estructural. En: Trastornos graves de la personalidad. Estrategias psicoterapéuticas. $1^{\mathrm{a}}$ ed. Editorial Manual Moderno. 1999 reimpresión. 